\title{
Preoperative Cetuximab, Irinotecan, Cisplatin, and Radiation Therapy for Patients With Locally Advanced Esophageal Cancer
}

\author{
Michael S. Lee, ${ }^{\text {a,h }}$ Harvey J. Mamon, ${ }^{c, h}$ Theodore S. Hong, ${ }^{\text {d,h }}$ Noah C. Chol, ${ }^{\text {d,h }}$ Panagiotis M. Fidias, ${ }^{\text {e, }}$ Eunice L. Kwak, ${ }^{e, h}$ \\ Jeffrey A. Meyerhardt, ${ }^{\text {g,h }}$ David P. Ryan, ${ }^{\text {e, h }}$ Raphael Bueno, ${ }^{\text {b,h }}$ Dean M. Donahue, ${ }^{\text {f,h }}$ Michael T. JakLitsch, ${ }^{\text {b,h }}$ Michael Lanuti, ${ }^{\text {f,h }}$ \\ David W. Ratther, ${ }^{\text {f,h }}$ Charles S. Fuchs, ${ }^{g, h}$ Peter C. Enzinger ${ }^{g, h}$ \\ aDepartment of Medicine and ${ }^{\mathrm{b}}$ Department of Surgery, Brigham and Women's Hospital, Boston, Massachusetts, USA; 'Department of \\ Radiation Oncology, Dana-Farber Cancer Institute and Brigham and Women's Hospital, Boston, Massachusetts, USA; ${ }^{\mathrm{d} D e p a r t m e n t}$ of \\ Radiation Oncology, ${ }^{e}$ Department of Medical Oncology, and ${ }^{\mathrm{f} D e p a r t m e n t}$ of Surgery, Massachusetts General Hospital, Boston, \\ Massachusetts, USA; ${ }^{\mathrm{g}}$ Department of Medical Oncology, Dana-Farber Cancer Institute, Boston, Massachusetts, USA; ${ }^{\mathrm{h}} \mathrm{Harvard}$ Medical \\ School, Boston, Massachusetts, USA \\ Disclosures of potential conflicts of interest may be found at the end of this article.
}

Key Words. Cetuximab • Esophageal cancer • EGFR • Chemoradiation

\section{AbSTRACT}

Purpose. To determine the efficacy and toxicity of weekly neoadjuvant cetuximab combined with irinotecan, cisplatin, and radiation therapy in patients with locally advanced esophageal or gastroesophageal junction cancer.

Methods and Materials. Patients with stage IIA-IVA esophageal or gastroesophageal junction cancer were enrolled in a Simon's two-stage phase II study. Patients received weekly cetuximab on weeks $0-8$ and irinotecan and cisplatin on weeks 1, 2, 4, and 5, with concurrent radiotherapy (50.4 Gy on weeks 1-6), followed by surgical resection.

Results. In the first stage, 17 patients were enrolled, 16 of whom had adenocarcinoma. Because of a low pathologic complete response ( $\mathrm{pCR}$ ) rate in this cohort, the trial was discontinued for patients with adenocarcinoma but squamous cell carcinoma patients continued to be enrolled; two addi- tional patients were enrolled before the study was closed as a result of poor accrual. Of the 19 patients enrolled, 18 patients proceeded to surgery, and 16 patients underwent an R0 resection. Three patients (16\%) had a pCR. The median progressionfree survival interval was 10 months, and the median overall survival duration was 31 months. Severe neutropenia occurred in $47 \%$ of patients, and severe diarrhea occurred in $47 \%$ of patients. One patient died preoperatively from sepsis, and one patient died prior to hospital discharge following surgical resection.

Conclusions. This schedule of cetuximab in combination with irinotecan, cisplatin, and radiation therapy was toxic and did not achieve a sufficient $\mathrm{pCR}$ rate in patients with localized esophageal adenocarcinoma to undergo further evaluation. The Oncologist 2013;18:281-287

Implications for Practice: Multimodality therapy, involving chemotherapy, radiotherapy, and surgery, has emerged as a standard treatment for locally advanced esophageal cancer. Prognosis with existing therapy for esophageal cancer remains poor, with a 5 -year survival rate of only $20 \%$ for locally advanced disease. Targeted therapies, such as EGFR inhibitors, are being evaluated for the treatment of locally advanced esophageal cancer, but remain investigational, with a number of ongoing randomized trials exploring the efficacy of various targeted therapies. This study showed that adding the EGFR inhibitor cetuximab to a preoperative chemoradiation regimen of irinotecan, cisplatin, and radiation therapy did not significantly improve rates of pathologic complete response at the time of surgery, and caused substantial toxicity.

\section{INTRODUCTION}

Traditionally, surgical resection has been one standard of care in the treatment of patients with locally advanced esophageal carcinoma. Based on the Radiation Therapy Oncology Group (RTOG) 8501 trial, combined chemoradiotherapy has been another standard of care for patients with locally advanced esophageal cancer. Preoperative chemoradiotherapy was found to be superior to surgery alone and is now an accepted standard treatment for patients with locally advanced esophageal carcinoma [1,2]. Irino- tecan and cisplatin combination therapy is an active, well-tolerated regimen for metastatic esophageal cancer [3], and it has been shown to be well tolerated and effective in combination with radiotherapy in patients with locally advanced esophageal cancer [4-6], with a median survival time of 25-30 months [4, 5].

Targeted therapy against the epidermal growth factor receptor (EGFR) may be an additional avenue of treatment for patients with esophageal carcinoma. EGFR is a protein ty-

Correspondence: Peter C. Enzinger, M.D., Dana-Farber Cancer Institute, 450 Brookline Avenue, Boston, Massachusetts 02215, USA. Telephone: 617-632-6855; Fax:617-632-5370; e-mail: peter_enzinger@dfci.harvard.edu Received May 17, 2012; accepted for publication September 28, 2012; first published online in The Oncologist Express on February 21, 2013. @AlphaMed Press 1083-7159/2013/\$20.00/0 http://dx.doi.org/ 10.1634/theoncologist.2012-0208 
rosine kinase that is overexpressed in a number of malignancies, including $32 \%-80 \%$ of esophageal adenocarcinomas [7, 8] and up to $80 \%$ of esophageal squamous cell carcinomas [9]. EGFR overexpression is associated with more advanced disease and a worse prognosis in patients with esophageal adenocarcinoma [8] and squamous cell carcinoma [9]. Cetuximab is a chimeric monoclonal antibody directed to the ligand-binding site of EGFR. When given with platinum-based chemotherapy, cetuximab resulted in a longer overall survival (OS) time in patients with recurrent or metastatic squamous cell head and neck carcinoma [10]. We therefore decided to design a phase II study adding cetuximab to irinotecan, cisplatin, and concurrent radiation therapy administered preoperatively in patients with advanced locoregional esophageal carcinoma.

\section{Methods And Materials}

\section{Eligibility Criteria}

Patients aged $\geq 18$ years with pathologically confirmed stage IIA, IIB, or III carcinoma of the esophagus or gastroesophageal junction (as defined by the American Joint Committee on Cancer [AJCC Cancer Staging Manual, 5th ed., http://www. cancerstaging.org/products/pasteditions.html]) and patients with stage IVA lower esophageal or gastroesophageal junction carcinoma with involved regional lymph nodes encompassed by the radiation field were eligible for this trial. These participants also had Eastern Cooperative Oncology Group (ECOG) performance status score of 0 or 1 . Required laboratory parameters for inclusion were an absolute neutrophil count $(A N C) \geq 1,500 / \mu \mathrm{L}$, a platelet count $\geq 100,000 / \mu \mathrm{L}$, a total bilirubin level $\leq 1.5 \mathrm{mg} / \mathrm{dL}$, creatinine $\leq 1.5 \mathrm{mg} / \mathrm{dL}$, serum glutamic oxaloacetic transaminase $\leq 2.5 \times$ the upper limit of normal (ULN), and alkaline phosphatase $\leq 5 \times$ the ULN. Exclusion criteria included any prior radiation therapy or chemotherapy, any prior surgery for esophageal carcinoma, and any prior treatment targeting the EGFR pathway. Patients with metastases to distant organs or nonregional lymph nodes or with biopsy-proven invasion into the tracheobronchial tree, tracheoesophageal fistula, or malignant pleural effusion, pericardial effusion, or ascites were ineligible. Individuals with another active malignancy or significant uncontrolled comorbidity making chemoradiation inadvisable were also ineligible. Patients with grade 2-4 diarrhea or peripheral neuropathy, with interstitial pulmonary fibrosis, with Gilbert's disease, with a seizure disorder on antiepileptic medications, or with a known allergy to murine proteins were excluded. All patients who were enrolled underwent staging studies including computed tomography (CT) scan of the chest, abdomen, and pelvis; positron emission tomography (PET); endoscopy with biopsy and endoscopic ultrasonography (EUS); CT scan or magnetic resonance imaging of the head; and bronchoscopy if the primary tumor was in the cervical or proximal thoracic esophagus.

\section{Treatment}

Patients received cetuximab weekly $\left(400-\mathrm{mg} / \mathrm{m}^{2}\right.$ loading dose on week 0 and $250 \mathrm{mg} / \mathrm{m}^{2}$ on weeks $1-8$ ). On weeks 1, 2, 4, and 5 , participants also received weekly doses of cisplatin (30 $\left.\mathrm{mg} / \mathrm{m}^{2}\right)$ followed by irinotecan $\left(65 \mathrm{mg} / \mathrm{m}^{2}\right)$ in the initial cohort of 17 patients. The total dose of radiation therapy was $50.4 \mathrm{~Gy}$ in $1.80 \mathrm{~Gy} /$ fraction per day given once daily for 5 days/week for 28 fractions over 5.6 weeks. A radiation beam energy of 6-15 MV photons was used. Treatment was given with a combination of anterior or posterior, oblique, and lateral fields such that the dose-to-target volume did not differ by $>10 \%$ from the dose specified at the isocenter. The gross tumor volume was defined by EUS, esophagram, or CT and included periesophageal lymph nodes measuring $>1.0 \mathrm{~cm}$ in short axis diameter. The clinical target volume was designated to extend $5 \mathrm{~cm}$ cranially and caudally and $2-3 \mathrm{~cm}$ radially beyond the gross tumor volume. The clinical target volume also included the medial supraclavicular fossa if the primary tumor was located above the carina, and a localized photon or electron boost to the supraclavicular fossa was allowed if the supraclavicular fossa dose was $<39.6 \mathrm{~Gy}$ at a 3-cm depth from the anterior skin surface. A barium swallow or PET-CT scan was used to confirm the location of the esophageal tumor. The spinal cord dose was limited to $45 \mathrm{~Gy}$, and the entire heart dose was limited to $30 \mathrm{~Gy}$, with $<50 \%$ of the heart receiving a maximum of $40 \mathrm{~Gy}$. No lung $>2 \mathrm{~cm}$ outside the target volume was allowed to receive a dose of $45 \mathrm{~Gy}$.

Esophagectomy was completed within 3-6 weeks after completing radiation therapy, and after repeat restaging evaluation, including at least a CT scan of the chest, abdomen, and pelvis, demonstrated no evidence of distant disease. The type of esophagectomy was at the discretion of the surgeon.

Four weeks after surgery, patients restarted cetuximab monotherapy $\left(250 \mathrm{mg} / \mathrm{m}^{2}\right.$ weekly) for 26 weeks.

\section{Assessments and Dose Modifications}

Toxicity was evaluated according to the National Cancer Institute's Common Toxicity Criteria (Version 1.0 http://www. ucdmc.ucdavis.edu/clinicaltrials/StudyTools/Documents/ NCl_Toxicity_Table.pdf) and the RTOG Radiation Morbidity Scoring Criteria (http://www.rtog.org/researchassociates/ adverseeventreporting/acuteradiationmorbidityscoringcriteria. aspx). If a patient experienced a grade 3 or 4 allergic or anaphylactic reaction to cetuximab, the patient was taken off study. Cetuximab was held for grade 3 or 4 skin toxicity, an ANC $<500 / \mathrm{mm}^{3}$, a platelet count $<50,000 / \mathrm{mm}^{3}$, and any grade 4 toxicity. Cisplatin was held for creatinine $>2.0 \mathrm{mg} / \mathrm{dl}$, grade 3 or 4 ototoxicity, and grade 3 or 4 neuropathy, and the dose was reduced by $50 \%$ for creatinine $>1.6 \mathrm{mg} / \mathrm{dL}$ and $\leq 2.0 \mathrm{mg} / \mathrm{dL}$. Irinotecan was held for an ANC $<1,000 / \mathrm{mm}^{3}$, a platelet count $<75,000 / \mathrm{mm}^{3}$, grade 3 or 4 diarrhea, or grade 4 fatigue, and the dose was subsequently reduced to $50 \mathrm{mg} / \mathrm{m}^{2}$. In addition, all nonhematologic grade 4 toxicities necessitated holding cisplatin, irinotecan, and cetuximab, and most grade 3 toxicities necessitated holding cisplatin and irinotecan. Radiation therapy was continued without interruption except for an $\mathrm{ANC}<1,000 / \mathrm{mm}^{3}$, a platelet count $<50,000 / \mathrm{mm}^{3}$, grade 3 esophagitis or mucositis, any grade 4 toxicity, or a 2-week consecutive hold of chemotherapy.

\section{Study Design}

We planned a Simon two-stage design, prospective, phase II study that initially enrolled 17 patients, with the plan to terminate the study if two or fewer pCRs were noted. If three or more pCRs were noted, an additional 18 patients were to be enrolled. With this design, the probability of terminating the study after enrolling 17 patients would be 0.76 if the true response rate was $10 \%$, but it would be 0.16 if the true response rate was $25 \%$. The primary endpoints were the $\mathrm{pCR}$ rate and 
Table 1. Patient characteristics

\begin{tabular}{lc}
\hline Characteristic & Value \\
\hline$n$ of patients & 19 \\
Median (range) age, yrs & $56(21-76)$ \\
Sex & $15(79 \%)$ \\
Male & $4(21 \%)$ \\
Female & \\
Eastern Cooperative Oncology Group & \\
performance status score & $7(37 \%)$ \\
0 & $12(63 \%)$ \\
1 & \\
Histology & $16(84 \%)$ \\
Adenocarcinoma & $3(16 \%)$ \\
Squamous cell carcinoma & \\
Tumor location & $2(11 \%)$ \\
Midesophagus & $5(26 \%)$ \\
Lower esophagus & $12(63 \%)$ \\
Gastroesophageal junction & \\
Clinical stage & $6(32 \%)$ \\
IIA & $1(5 \%)$ \\
IIB & $8(42 \%)$ \\
III & $4(21 \%)$ \\
\hline
\end{tabular}

toxicity with this regimen. Secondary endpoints were the median progression-free survival (PFS) and median OS times. The study was approved by the institutional review board of the Dana-Farber Harvard Cancer Center, and all patients enrolled provided informed consent for participation.

\section{RESULTS}

\section{Study Design Modification}

Seventeen patients were initially enrolled in the study, 16 of whom had adenocarcinoma. The PCR rate did not meet the prespecified criteria to enroll more patients with adenocarcinoma. However, given the low enrollment of patients with squamous cell carcinoma, approval was obtained from the institutional review board to continue enrollment of patients with squamous cell carcinoma, with the starting dose of irinotecan decreased to $50 \mathrm{mg} / \mathrm{m}^{2}$, given the rate of adverse events in the original cohort. An additional two patients were enrolled before the study was closed as a result of poor accrual of this subgroup of patients.

\section{Patient Characteristics}

In total, 19 patients were enrolled from July 2004 to July 2007 (Table 1). The median age at enrollment was 56 years. The majority of patients were male $(79 \%)$ and had adenocarcinoma $(84 \%)$. The most common sites of primary tumor were the gastroesophageal junction (63\%) and the lower esophagus (26\%). At the time of presentation, $31 \%$ of patients were clinical AJCC stage IIA, $5 \%$ of patients were stage IIB, $42 \%$ of patients were stage III, and $21 \%$ of patients were stage IVA.

\section{Toxicity}

The most common hematologic grade 3 or 4 adverse event preoperatively was neutropenia (47\%), including febrile neu-
Table 2. Most common preoperative grade 3 or 4 adverse events $(n=19)$

\begin{tabular}{|c|c|c|}
\hline Adverse event & Grade 3 & Grade 4 \\
\hline \multicolumn{3}{|l|}{ Hematologic } \\
\hline Neutrophils & $6(32 \%)$ & $3(16 \%)$ \\
\hline Platelets & $1(5 \%)$ & 0 \\
\hline \multicolumn{3}{|l|}{ Nonhematologic } \\
\hline \multicolumn{3}{|l|}{ Gastrointestinal } \\
\hline Anorexia & $3(16 \%)$ & $2(11 \%)$ \\
\hline Constipation & $2(11 \%)$ & 0 \\
\hline Dehydration & $6(32 \%)$ & 0 \\
\hline Diarrhea & $9(47 \%)$ & 0 \\
\hline Dysphagia, esophagitis, or odynophagia & $5(26 \%)$ & $1(5 \%)$ \\
\hline Nausea & $6(32 \%)$ & 0 \\
\hline Vomiting & $4(21 \%)$ & 0 \\
\hline \multicolumn{3}{|l|}{ Systemic } \\
\hline Fatigue & $2(11 \%)$ & $1(5 \%)$ \\
\hline Hypotension & $2(11 \%)$ & $1(5 \%)$ \\
\hline Infection with neutropenia & $2(11 \%)$ & 0 \\
\hline Infection without neutropenia & 0 & $1(5 \%)$ \\
\hline Neutropenic fever & $4(21 \%)$ & $1(5 \%)$ \\
\hline \multicolumn{3}{|l|}{ Cetuximab-related } \\
\hline Allergic or anaphylactic reaction & 0 & 0 \\
\hline Rash or desquamation & $1(5 \%)$ & 0 \\
\hline
\end{tabular}

Each event represents one patient who developed the toxicity, recorded here at the highest grade of toxicity noted in the individual patient.

tropenia in $26 \%$ of patients (Table 2 ). The most common nonhematologic grade 3 or 4 adverse events were diarrhea (47\%), dehydration (31\%), dysphagia or esophagitis (31\%), nausea (31\%), anorexia (26\%), and vomiting (21\%). One patient died as a result of Aspergillus infection causing respiratory failure and sepsis prior to surgery. Two patients were withdrawn from the study on weeks $5-6$ because of grade 3 or 4 toxicity, but both patients completed courses of cisplatin and irinotecan and proceeded to surgery off study, and their data are included for analysis. No patients had an allergic or anaphylactic reaction to cetuximab. Only one patient suffered a grade 3 rash, though 17 patients developed a grade 1 or 2 rash.

Nine patients required irinotecan dose reductions. Two patients required cetuximab dose reductions. Radiation therapy needed to be delayed at least once for 14 patients.

Of the 19 patients enrolled, 18 proceeded to surgery and 16 successfully underwent an $\mathrm{R} 0$ surgical resection of their primary tumor. The remaining two patients were found to have progressed and their surgeries were aborted without resection. The median duration of admission for surgery was 12.5 days (range, 8-49 days), with four patients hospitalized for $>14$ days. One patient died after a 49-day hospitalization with persistent respiratory failure. No patient developed anastomotic leak. Other major complications included chylothorax requiring surgery or embolization in two patients, reintubation for respiratory failure in three patients, postoperative myocardial infarction in one patient, and small bowel obstruction in one patient. Grade 3 or 4 toxicities that arose after surgery included pneumonitis or respiratory failure in three 

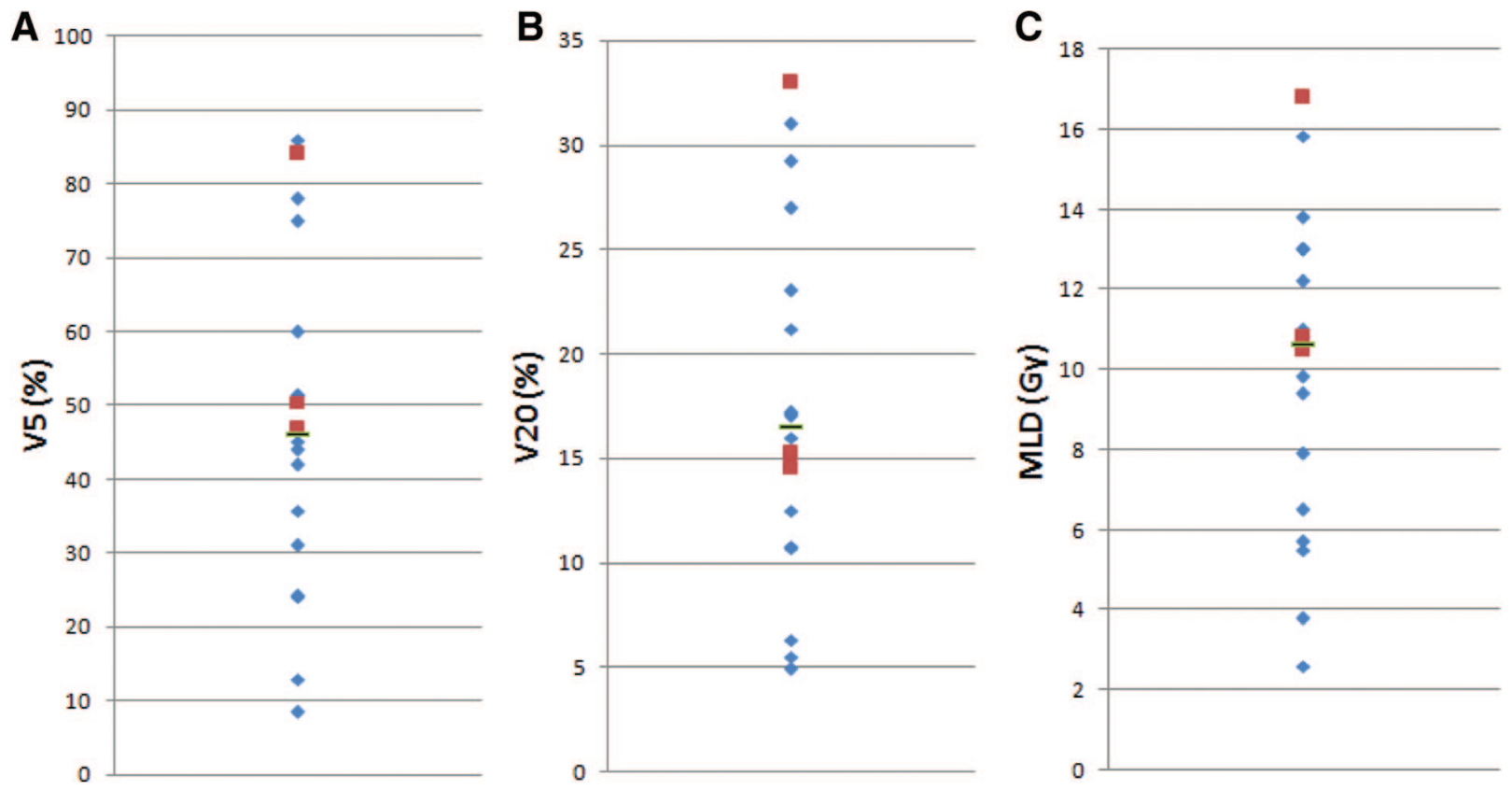

Figure 1. Dose-volume histogram data for 18 patients, including percentage of irradiated volume $>5 \mathrm{~Gy}$ (V5) (A), percentage of irradiated volume $>20 \mathrm{~Gy}(\mathrm{~V} 20)$ (B), and mean lung dose (MLD) (C). Indicated are the median (black bar), along with individual values for the 15 patients who did not have postoperative respiratory failure (blue diamonds) and the three patients who did have postoperative respiratory failure (red squares).

patients, hypomagnesemia in two patients, infection without neutropenia in two patients, and nausea in one patient.

Dose-volume histogram parameters of the lung, including the mean lung dose (MLD) and percentage of irradiated volume $>20$ Gy (V20) and >5 Gy (V5), were determined for 18 patients, including all three patients who developed respiratory failure (Fig. 1). The median V5 was $46.0 \%$ (range, 8.5\%$86 \%)$; for the three patients with respiratory failure, the V5 was $46.8 \%, 50.5 \%$, and $84 \%$. The median V20 was $16.5 \%$ (range, $4.9 \%-33 \%$ ); for the three patients with respiratory failure, the $V 20$ was $14.5 \%, 15.2 \%$, and $33 \%$. The median MLD was $10.7 \mathrm{~Gy}$ (range, 2.6-16.8 Gy); the MLDs for the three patients with respiratory failure were $10.5 \mathrm{~Gy}, 10.8 \mathrm{~Gy}$, and $16.8 \mathrm{~Gy}$.

Of the 16 patients who underwent an R0 resection, 11 received at least one postoperative dose of cetuximab. Eight patients completed at least half of their postoperative course of cetuximab and five patients completed the entire 26-week postoperative course.

\section{pCR and Survival Outcomes}

Sixteen of 19 patients underwent an R0 surgical resection: seven received three-hole esophagectomy, five received IvorLewis esophagectomy, three received left thoracoabdominal esophagectomy, and one received minimally invasive esophagectomy. Of the remaining three patients, one died preoperatively as a result of sepsis and two had unresectable disease. For all 19 patients, the median PFS interval was 10 months, with a range of 2 months to $>88$ months, and the median OS time was 31 months, with a range of 2 months to $>88$ months. Three of the 19 patients (16\%) achieved a pCR. With a minimum follow-up duration of 67 months, five patients $(26 \%)$ remained alive and 14 patients had died. Three patients continued in remission.

Of the 16 patients enrolled with adenocarcinoma, 13 underwent an R0 resection of their primary tumor. Of these 13 patients, one had a pCR, eight had a partial response (including one with only residual carcinoma in situ), three had stable disease, and one had progression. The median PFS interval was 9 months (range, 2 months to $>88$ months) and the median OS time was 19.5 months (range, 2 months to $>88$ months).

Of the three patients enrolled with squamous cell carcinoma, all proceeded to an R0 surgical resection. Two of the three patients experienced a pCR and the third patient had stable disease. The PFS times were 25 months, 38 months, and $>88$ months. The OS times were 48 months, $>67$ months, and $>88$ months.

\section{DisCUSSION}

This study demonstrated that preoperative cetuximab, irinotecan, cisplatin, and concurrent radiation therapy in patients with locally advanced esophageal cancer is toxic and did not achieve a sufficient $\mathrm{pCR}$ rate to undergo further investigation in patients with adenocarcinoma. Unfortunately, there were too few patients to evaluate the efficacy of the regimen in patients with squamous cell carcinoma. Overall, only three patients $(16 \%)$ achieved a pCR. Notably, there were high rates of grade 3 or 4 toxicity, including neutropenia (47\%) and diarrhea (47\%). Rates of $p C R$ of $25 \%-40 \%$ have been reported in previous studies assessing trimodality therapy with preoperative chemoradiation [1]. Compared with this standard, this regimen was not considered promising for further development.

Several trials have investigated the use of irinotecan, cisplatin, and radiation therapy without the addition of targeted biologic agents in patients with locally advanced esophageal cancer. Compared with those trials, our regimen was more toxic and associated with a lower $\mathrm{pCR}$ rate $[4-6,11,12]$ (Table 3). Those trials enrolled similar numbers of adenocarcinoma patients $(70 \%-100 \%)$ and reported pCR rates of $15 \%-33 \%$. The median OS time was in the range of 25-36 months. Our tri- 
Table 3. Comparison of trials

\begin{tabular}{|c|c|c|c|c|c|}
\hline Study & $\begin{array}{l}n \text { of patients } \\
\text { (\% adenocarcinoma: } \\
\% \text { SCC) }\end{array}$ & Chemotherapy & ITT pCR & $\begin{array}{l}\text { OS, } \\
\text { mos }\end{array}$ & $\begin{array}{l}\text { Grade } 3-4 \text { toxicities ( } \geq 15 \% \text { ) and grade } 5 \\
\text { toxicities }\end{array}$ \\
\hline \multicolumn{6}{|c|}{$\begin{array}{l}\text { Cisplatin, irinotecan, and radiation therapy } \\
\text { regimens }\end{array}$} \\
\hline Ilson et al. [4] & $19(84: 16)$ & Cisplatin, irinotecan, and XRT & $21 \%$ & 25 & Neutropenia, $21 \%$; thrombosis, $21 \%$ \\
\hline Knox et al. [5] & $52(75: 25)$ & Cisplatin, irinotecan, and XRT & $13 \%$ & 36 & $\begin{array}{l}\text { Neutropenia, } 36 \% \text {; esophagitis/dysphagia, } \\
16 \% \text {; fatigue, } 16 \% \text {; stroke/death, } 2 \%\end{array}$ \\
\hline Enzinger et al. [6] & $40(85: 15)$ & $\begin{array}{l}\text { Cisplatin, irinotecan, celecoxib, } \\
\text { and XRT }\end{array}$ & $33 \%$ & 34 & $\begin{array}{l}\text { Neutropenia, } 22 \% \text {; diarrhea/dehydration, } \\
15 \% \text {; ARDS/death, } 2.5 \%\end{array}$ \\
\hline Tew et al. [11] & $41(76: 24)$ & Cisplatin, irinotecan, and XRT & $16 \%$ & NR & Neutropenia, $37 \%$; thrombosis, $15 \%$ \\
\hline Kleinberg et al. [12] & $46(100: 0)$ & Cisplatin, irinotecan, and XRT & $15 \%$ & 34.9 & Hematologic, $43 \%$ \\
\hline \multicolumn{6}{|c|}{$\begin{array}{l}\text { Cisplatin, irinotecan, and radiation therapy } \\
\text { regimens with cetuximab }\end{array}$} \\
\hline This study & $19(84: 16)$ & $\begin{array}{l}\text { Cisplatin, irinotecan, cetuximab, } \\
\text { and XRT }\end{array}$ & $16 \%$ & 31 & $\begin{array}{l}\text { Neutropenia (47\%); diarrhea, } 47 \% \text {; } \\
\text { dehydration, 31\%; dysphagia/esophagitis, } \\
\text { 31\%; nausea, 31\%; anorexia, 26\%; } \\
\text { vomiting, } 21 \% \text {; sepsis/death, 5\%; } \\
\text { respiratory failure/death, } 5 \%\end{array}$ \\
\hline Tomblyn et al. [13] & $21(48: 52)$ & $\begin{array}{l}\text { Cisplatin, irinotecan, cetuximab, } \\
\text { and XRT }\end{array}$ & NR & 11.2 & $\begin{array}{l}\text { Hematologic, } 54 \% \text {; fatigue, } 24 \% \text {; diarrhea, } \\
24 \% \text {; nausea/vomiting, } 19 \% \text {; dehydration, } \\
19 \% \text {; anorexia, } 19 \% \text {; sudden death, } 5 \% \text {; } \\
\text { gastrointestinal necrosis/death, } 5 \%\end{array}$ \\
\hline \multicolumn{6}{|c|}{$\begin{array}{l}\text { Other chemoradiotherapy regimens with } \\
\text { cetuximab }\end{array}$} \\
\hline Lledo et al. [14] & $79(33: 67)$ & $\begin{array}{l}\text { Oxaliplatin, 5-FU, LV, cetuximab, } \\
\text { and XRT }\end{array}$ & NR & NR & Neutropenia, $28 \%$ \\
\hline Kleinberg et al. [15] & $22(100: 0)$ & $\begin{array}{l}\text { Oxaliplatin, 5-FU, cetuximab, and } \\
\text { XRT }\end{array}$ & $32 \%$ & NR & $\begin{array}{l}\text { ARDS/death, } 18 \% \text {; PE/death, 5\%; sepsis/ } \\
\text { death, } 5 \%\end{array}$ \\
\hline Safran et al. [17] & $60(80: 20)$ & $\begin{array}{l}\text { Carboplatin, paclitaxel, cetuximab, } \\
\text { and XRT }\end{array}$ & $25 \%$ & NR & $\begin{array}{l}\text { Rash, } 25 \% \text {; esophagitis, } 16 \% \text {; dehydration, } \\
16 \%\end{array}$ \\
\hline Ruhstaller et al. [18] & $28(54: 46)$ & $\begin{array}{l}\text { Cisplatin, docetaxel, cetuximab, } \\
\text { and XRT }\end{array}$ & $32 \%$ & NR & $\begin{array}{l}\text { Febrile neutropenia, } 21 \% \text {; } \\
\text { dysphagia/esophagitis, } 26 \%\end{array}$ \\
\hline
\end{tabular}

For this table, poorly differentiated or undifferentiated cases were added to the adenocarcinoma totals.

aITT calculation of pCR calculated as $\mathrm{pCR} /$ (all patients who received therapy). Of note, some studies did not require patients to proceed to surgery (Ilson et al. [4], Safran et al. [17]).

Abbreviations: 5-FU, 5-fluorouracil; ARDS, acute respiratory distress syndrome; ITT, intention to treat; LV, leucovorin; NR, not reported; pCR,

pathologic complete response; $\mathrm{PE}$, pulmonary embolism; SCC, squamous cell carcinoma; XRT, radiation therapy.

al's PCR of $16 \%$ and median OS duration of 31 months falls within the range of those studies, but the addition of cetuximab to the regimen did not appear to improve these measures of efficacy. Furthermore, there was substantial toxicity with our regimen, with grade 3 or 4 neutropenia in $47 \%$ of patients and grade 3 or 4 diarrhea in $47 \%$ of patients. In the other trials, severe neutropenia occurred in $21 \%-37 \%$ of patients and severe diarrhea occurred in $<15 \%$ of patients.

Results similar to ours were reported recently by the Southwest Oncology Group (S0414 trial), who investigated the role of cetuximab, irinotecan, cisplatin, and thoracic radiotherapy in patients with unresectable esophageal carcinoma [13]. That trial enrolled 21 eligible patients and achieved a median PFS time of 6.4 months and median OS time of 11.2 months. Toxicities were high, with two deaths on protocol and high rates of grade 3 or 4 hematologic toxicity (52\%), diarrhea (24\%), fatigue (24\%), nausea or emesis (19\%), anorexia (19\%), and dehydration (19\%). This provides further evidence that cetuximab in combination with irinotecan, cisplatin, and radiation therapy results in substantial toxicity.

However, other chemoradiation combinations with cetuximab may be more promising. Two chemoradiation studies have used cetuximab in combination with oxaliplatin and fluorouracil, with conflicting results regarding the safety of this regimen. Lledo and colleagues combined cetuximab with fluorouracil, leucovorin, and oxaliplatin plus radiation therapy and found an overall clinical response rate of $77.2 \%$ and a clinical complete response rate of $40.5 \%$ without reports of postoperative pneumonitis or acute respiratory distress syndrome (ARDS) [14]. Kleinberg and colleagues used cetuximab in combination with neoadjuvant oxaliplatin, fluorouracil, and radiation therapy, followed by surgery and adjuvant cetuximab and docetaxel, and found a $32 \%$ pCR rate [15]. However, four of 22 patients died postoperatively as a result of ARDS, and a total of seven deaths occurred on study; enrollment was halted. There was no clear relationship between ARDS and radiotherapy dose; patients who developed ARDS had a V20 of $17 \%-19 \%$ (median V20, 11\%) and a V 5 of $46 \%-72 \%$ (median V5, 50\%) [15]. Similarly, in our study, though the patient with the highest V5, V20, and MLD did suffer respiratory failure, the other two patients who developed respiratory failure had values close to the median for all three parameters. However, all three patients with respiratory failure did have a V5 greater than the median, and there is correlation between the lung volume spared from $\geq 5$ Gy radiation and a lower risk for postoperative pulmonary complications in patients with esophageal cancer treated with concurrent chemoradiotherapy [16]. The risk for postoperative pulmonary toxicity remains worrisome. Though Lledo and colleagues did not find a significant rate of ARDS in their trial, only $25 \%$ of patients underwent surgical resection of their primary tumor after chemoradiotherapy. A planned French 
phase III study comparing cetuximab, oxaliplatin, fluorouracil, and radiation therapy with standard cisplatin, fluorouracil, and radiation therapy may help shed further light on this issue.

Other studies have combined cetuximab with a taxane, platinum analog, and radiation therapy. Safran and colleagues treated 60 patients with cetuximab, paclitaxel, carboplatin, and radiation therapy [17]. That regimen achieved a $27 \%$ pCR rate in patients who proceeded to surgery. Toxicities were lower than in our study, with grade 3 or 4 toxicities of rash (25\%), esophagitis (16\%), dehydration (16\%), neutropenia (14\%), and anemia (11\%) being most common. The median survival time was not reported. Ruhstaller and colleagues treated 28 patients with cetuximab, docetaxel, cisplatin, and radiation therapy [18]. Thirty-two percent of those patients had a pCR, and an additional $36 \%$ of patients had microscopic residual disease. At 12 months, the event-free survival rate was $82 \%$ and OS rate was $86 \%$. In our study, the 12 -month event-free survival rate was $42 \%$ and OS rate was $58 \%$. Based on the promising results of these trials, phase III trials have been initiated. The RTOG 0436 trial is randomizing patients to receive definitive nonoperative treatment with paclitaxel, cisplatin, and radiation with or without cetuximab. The Swiss Group for Clinical Cancer Research (SAKK) 75/08 trial is similarly randomizing patients to receive treatment with docetaxel, cisplatin, and radiation with or without cetuximab.

In our study, the relatively low pCR rate may be associated with the high toxicity of the regimen necessitating delays and dose attenuations in the chemotherapy and radiation therapy that may have reduced the efficacy of this regimen. It is unlikely that there is an intrinsic difference in the efficacy of a regimen based on a taxane-platinum combination versus an irinotecanplatinum combination, because the ECOG 1201 trial compared the efficacy of neoadjuvant cisplatin, irinotecan, and radiation therapy with that of neoadjuvant cisplatin, paclitaxel, and radiation therapy and found comparable pCR rates (15\% vs. $16 \%$, respectively) [13] and comparable survival outcomes [19].

The role of anti-EGFR therapy, particularly with antibodies, in patients with esophagogastric cancer may now be uncertain based on the results of the Randomized Trial of Epirubicin, Oxaliplatin, and Capecitabine With or Without Panitumumab for Advanced and Locally Advanced Esophagogastric Cancer (REAL-3) study recently presented at the American Society of Clinical Oncology symposium in Chicago [20]. That randomized multicenter study of standard chemotherapy with or without the EGFR antibody panitumumab in patients with chemotherapy-naïve advanced esophagogastric adenocarcinoma demonstrated no survival benefit with the addition of the EGFR antibody. In fact, patients who received panitumumab had a worse OS outcome than patients who did not receive the antibody ( 8.8 months vs. 11.3 months; hazard ratio, $1.37 ; 95 \%$ confidence interval, $1.07-1.76 ; p=.013$ ). The reason for this lack of efficacy is unclear, and it may not neces- sarily translate into the neoadjuvant setting, where chemotherapy primarily acts as a sensitizer to radiation. The authors of the REAL-3 study analyzed a subgroup of patients for KRAS and PIK3CA mutations and the expression of either phosphatase and tensin homologue deleted on chromosome ten (PTEN) or human epidermal growth factor receptor (HER)-2. In a multivariate analysis, KRAS and PIK3CA mutation, but not PTEN or HER-2 expression, appeared to be prognostic. Others have suggested that MET amplification (7.1 months vs. 16.2 months; $p<.001$ ) and EGFR amplification (11.2 months vs. 16.2 months; $p=.16$ ) may have a negative impact on survival outcomes in patients with esophagogastric cancer [21]. Activating mutations within EGFR have also been identified [22]. Thus, other irregularities in the EGF pathway or deviations in other associated pathways could explain the potential resistance of esophagogastric cancer cells to anti-EGFR therapy. In summary, although our study and the S0414 study showed that cetuximab with irinotecan, cisplatin, and radiation therapy yielded low efficacy and high toxicity, other chemoradiotherapy combinations with cetuximab appear more promising. Ultimately, only a randomized comparison will determine the role of cetuximab in the treatment of patients with locally advanced esophageal cancer. Enrollment in the ongoing phase III RTOG 0436 and SAKK $75 / 08$ studies is strongly encouraged.

\section{ACKNOWLEDGMENTS}

Supported by Bristol-Myers-Squibb and the National Institutes of Health (grant P50-CA127003).

Presented in part at the 42nd Annual Meeting of the American Society of Clinical Oncology, Atlanta, GA, June 2-6, 2006

\section{Author Contributions \\ Conception/Design: Harvey J. Mamon, Charles Fuchs, Peter C. Enzinger \\ Provision of study material or patients: Harvey J. Mamon, Theodore S. Hong, Noah C. Choi, Panagiotis M. Fidias, Eunice L. Kwak, Jeffrey A. Meyerhardt, David P. Ryan, Raphael Bueno, Dean M. Donahue, Michael T. Jaklitsch, Mi- chael Lanuti, David W. Rattner, Charles Fuchs, Peter C. Enzinger \\ Collection and/or assembly of data: Michael S. Lee, Harvey J. Mamon, Peter C. Enzinger \\ Data analysis and interpretation: Michael S. Lee, Theodore S. Hong, Peter C. Enzinger \\ Manuscript writing: Michael S. Lee, Peter C. Enzinger \\ Final approval of manuscript: Michael S. Lee, Harvey J. Mamon, Theodore S. Hong, Noah C. Choi, Panagiotis M. Fidias, Eunice L. Kwak, Jeffrey A. Meyer hardt, David P. Ryan, Raphael Bueno, Dean M. Donahue, Michael T. Jaklitsch, Michael Lanuti, David W. Rattner, Charles Fuchs, Peter C. Enzinger}

\section{DISCLOSURES}

Theodore S. Hong: Illumina (C/A); Novartis (RF); Panagiotis M. Fidias:

Genentech (C/A); Raphael Bueno: Exosome Inc. (C/A); Covidien Inc.

Device Company (H); Castle Bioscience (RF); David W. Rattner:

Olympus Medical (C/A); Charles S. Fuchs: Bristol Myers Squibb, Amgen, Genentech, Metamark Genetics, Sanofi, Bayer (C/A); Peter C. Enzinger: Genentech/Roche, Sanofi-Aventis, Boehringer-Ingelheim (C/A); Other: Data safety monitoring board: Taiho.

The other authors indicated no financial relationships.

(C/A) Consulting/advisory relationship; (RF) Research funding; (E) Employment; (H) Honoraria

received; (OI) Ownership interests; (IP) Intellectual property rights/inventor/patent holder; (SAB) Scientific advisory board

\section{REFERENCES}

1. Gebski V, Burmeister B, Smithers BM et al. Survival benefits from neoadjuvant chemoradiotherapy or chemotherapy in oesophageal carcinoma: A meta-analysis. Lancet Oncol 2007;8:226-234.

2. Van der Gaast $A$, van Hagen $P$, Hulshof $M$ et al. Effect of preoperative concurrent chemoradiotherapy on survival of patients with resectable esophageal or esophagogastric junction cancer: Results from a multicenter randomized phase III study. J Clin Oncol 2010;28(suppl 15):abstract 4004

3. Ilson DH, Saltz L, Enzinger P et al. Phase II trial of weekly irinotecan plus cisplatin in advanced esophageal cancer. J Clin Oncol 1999;17:3270-3275.
4. Ilson DH, Bains M, Kelsen DP et al. Phase I trial of escalating-dose irinotecan given weekly with cisplatin and concurrent radiotherapy in locally advanced esophageal cancer. J Clin Oncol 2003;21:29262932.

5. Knox JJ, Wong R, Visbal AL et al. Phase 2 trial of preoperative irinotecan plus cisplatin and confor- 
mal radiotherapy, followed by surgery for esophageal cancer. Cancer 2010;116:4023-4032.

6. Enzinger PC, Mamon $\mathrm{H}$, Choi $\mathrm{N}$ et al. Phase II cisplatin, irinotecan, celecoxib and concurrent radiation therapy followed by surgery for locally advanced esophageal cancer. Presented at the 2004 Gastrointestinal Cancers Symposium; January 22-24, 2004; San Francisco, CA

7. Cronin J, McAdam E, Danikas A et al. Epidermal growth factor receptor (EGFR) is overexpressed in high-grade dysplasia and adenocarcinoma of the esophagus and may represent a biomarker of histological progression in Barrett's esophagus (BE). Am J Gastroenterol 2011;106:46-56.

8. Wang KL, Wu TT, Choi IS et al. Expression of epidermal growth factor receptor in esophageal and esophagogastric junction adenocarcinomas: Association with poor outcome. Cancer 2007;109:658667.

9. Itakura $Y$, Sasano $H$, Shiga $C$ et al. Epidermal growth factor receptor overexpression in esophageal carcinoma. An immunohistochemical study correlated with clinicopathologic findings and DNA amplification. Cancer 1994;74:795-804.

10. Vermorken JB, Mesia R, Rivera F et al. Platinum-based chemotherapy plus cetuximab in head and neck cancer. N Engl J Med 2008;359:11161127.

11. Tew WP, Minsky B, Bains M et al. Phase II trial of preoperative combined modality therapy for esophageal carcinoma: Induction cisplatin-irinotecan followed by concurrent cisplatin-irinotecan and radiotherapy. J Clin Oncol 2005;23(suppl 16):abstract 4017.

12. Kleinberg L, Powell ME, Forastiere A et al. E1201: An Eastern Cooperative Oncology Group (ECOG) randomized phase II trial of neoadjuvant preoperative paclitaxel/cisplatin/RT or irinotecan/ cisplatin/RT in endoscopy with ultrasound (EUS) staged adenocarcinoma of the esophagus. J Clin Oncol 2007;25(suppl 18):abstract 4533.

13. Tomblyn MB, Goldman BH, Thomas CR Jr et al. Cetuximab plus cisplatin, irinotecan, and thoracic radiotherapy as definitive treatment for locally advanced, unresectable esophageal cancer: A phase-II study of the SWOG (S0414). J Thorac Oncol 2012;7: 906-912.

14. Lledo G, Michel P, Dahan L et al. Chemoradiation with FOLFOX plus cetuximab in locally advanced cardia or esophageal cancer: Final results of a GERCOR phase II trial (ERaFOX). J Clin Oncol 2011; 29(suppl 4):abstract 8.

15. Kleinberg LR, Catalano PJ, Gibson MK et al. ECOG 2205: A phase II study to measure response rate and toxicity of neo-adjuvant chemoradiotherapy (CRT) (IMRT permitted) with oxaliplatin (O) and infusional 5-fluorouracil (5FU) plus cetuximab (C225) in patients with operable adenocarcinoma of the esophagus: High risk of post-op adult respiratory distress syndrome (ARDS). Int J Radiat Oncol Biol Phys 2010;78:S72.

16. Wang SL, Liao Z, Vaporciyan AA et al. Investigation of clinical and dosimetric factors associated with postoperative pulmonary complications in esophageal cancer patients treated with concur- rent chemoradiotherapy followed by surgery. Int J Radiat Oncol Biol Phys 2006;64:692-699.

17. Safran $H$, Suntharalingam M, Dipetrillo T et al. Cetuximab with concurrent chemoradiation for esophagogastric cancer: Assessment of toxicity. Int J Radiat Oncol Biol Phys 2008;70:391-395.

18. Ruhstaller T, Pless M, Dietrich D et al. Cetuximab in combination with chemoradiotherapy before surgery in patients with resectable, locally advanced esophageal carcinoma: A prospective, multicenter phase IB/II trial (SAKK 75/06). J Clin Oncol 2011;29:626-631.

19. Kleinberg $L$, Powell ME, Forastiere AA et al. Survival outcome of E1201: An Eastern Cooperative Oncology Group (ECOG) randomized phase II trial of neoadjuvant preoperative paclitaxel/cisplatin/radiotherapy (RT) or irinotecan/cisplatin/RT in endoscopy with ultrasound (EUS) staged esophageal adenocarcinoma. J Clin Oncol 2008;26(suppl 15): abstract 4532

20. Waddell T, Chau I, Barbachano Y et al. A randomized multicenter trial of epirubicin, oxaliplatin and capecitabine (EOC) plus panitumumab in advanced esophagogastric cancer (REAL3). J Clin Oncol 2012;30(suppl 15):abstract LBA4000.

21. Lennerz JK, Kwak EL, Ackerman A et al. MET amplification identifies a small and aggressive subgroup of esophagogastric adenocarcinoma with evidence of responsiveness to crizotinib. J Clin Oncol 2011;29:4803-4810.

22. Kwak EL, Jankowski J, Thayer SP et al. Epidermal growth factor receptor kinase domain mutations in esophageal and pancreatic adenocarcinomas. Clin Cancer Res 2006;12:4283-4287. 